\title{
Ein Unscented Kalman Filter zur Schätzung von Schaltungsnichtidealitäten eines zeitkontinuierlichen Sigma-Delta Wandlers mit impliziter Dezimation
}

\author{
A. Buhmann, M. Keller, M. Maurer, M. Ortmanns, and Y. Manoli \\ Universität Freiburg - IMTEK, Lehrstuhl für Mikroelektronik, Georges-Koehler-Allee 102, 79110 Freiburg, Germany
}

\begin{abstract}
Zusammenfassung. Nichtidealitäten einer Schaltung, wie z.B. nicht ideale Charakteristik des Operationsverstärkers und Streuungen in den Filterkoeffizienten, sind dahingehend bekannt die Effizienz von zeitkontinuierlichen Sigma-Delta Wandlern in drastischer Weise zu reduzieren. Daher stellt diese Veröffentlichung eine mögliche Methode vor, um die genannten Nichtidealitäten durch eine Schätzung mit Hilfe eines Unscented Kalman Filters zu bestimmen und in einem möglichen weiteren Schritt zu korrigieren. Des Weiteren kann durch eine leichte Modifikation des vorgestellten Algorithmus auch gleichzeitig eine implizite Dezimation des Ausgangssignals durchgeführt werden. Hierdurch wird die Gesamteffizienz des vorgestellten Ansatzes gesteigert, da kein zusätzlicher Dezimationsfilter mehr benötigt wird. Simulationsergebnisse des Filteralgorithmus zeigen die prinzipielle Funktion des Algorithmus.
\end{abstract}

\section{Einleitung}

Schaltungsnichtidealitäten von zeitkontinuierlichen SigmaDelta Wandlern, wie z.B. nichtideales Verhalten des Operationsverstärkers, Streuung in den Filterkoeffizienten, ExcessLoop-Delay und Jitter, beeinträchtigen im großem Maße die Effizienz von Analog/Digital Wandler (ADC). Dies geschieht wegen der benötigten Skalierung des Schleifenfilters um unter der genannten Variation ein stabiles System zu erhalten. Zwar ermöglicht das Skalieren der Filterkoeffizienten ein stabiles System, aber dies wird mit einer geringeren Effizienz im Vergleich zum unskalierten Modulator erkauft.

Um diesen Nachteil zu umgehen, werden in der Literatur verschiedene Methoden vorgeschlagen. Diese lassen sich hauptsächlich in zwei Gruppen bezüglich ihres Funktionsprinzips unterteilen. Die erste Gruppe verwendet ei-

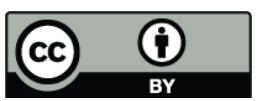

Correspondence to: A. Buhmann (buhmann@imtek.de) ne Offline Methode, um verschiedene Nichtidealitäten, wie z.B. endliche Bandbreite, Variation der Filterkoeffizienten und Verstärkung, wie auch spektrale Störung, zu schätzen (Cauwenberghs and Temes, 2000; Balestrieri et al., 2004). Hierzu wird der Eingang zum Wandler unterbrochen und durch ein bekanntes analoges Signal ersetzt. Die linearen Fehler der analogen Schaltung werden dann durch einen digitalen Filter korrigiert, indem das sich ergebende Regressionsproblem gelöst wird, oder indem der analoge Schaltkreis abgestimmt wird. Dieser Ansatz weist aber den erheblichen Nachteil auf, dass hierzu das Eingangssignal abgeklemmt werden muss, was leider nicht immer möglich ist. Des Weiteren ist eine online Kalibrierung wünschenswert, um auch beispielsweise Temperaturschwankungen und Alterung der Schaltung kompensieren zu können. Diese zusätzliche Anforderung an die Kalibrierungsmethode führt direkt zu der zweiten Gruppe, welche eine online Korrektur des ADCs ermöglichen. Bei diesen Verfahren wird ein bekanntes breitbandiges Signal dem eigentlichen Eingangssignal überlagert (Cauwenberghs and Temes, 2000). Die Fehler des ADCs werden dann durch eine mathematische Korrelation der Eingang und Ausgangssignals geschätzt. Die Korrektur kann durch Kalibrierung oder digitale Filterung erreicht werden. Ein weiterer Ansatz ist eine referenzfreie online Kalibrierung. Bei dieser wird zum Eingang des Quantisierers ein bekanntes Zufallszahl hinzu addiert. Auch dann kann in einem zweiten Schritt der Fehler durch Kalibrierung oder digitale Filterung reduziert werden. Alle diese Methoden weisen aber den großen Nachteil auf, dass nur kleine Abweichungen korrigiert werden können. Dies liegt an der endlichen Reihenentwicklung, welche für die Korrelation verwendet wird. Das Problem lässt sich aber nun umgehen, indem ein Unscented Kalman Filter (UKF) zur Schätzung der Abweichungen verwendet wird. Dadurch lassen sich die Zustandsvariablen und die unbekannten Parameter schätzen (Buhmann et al., 2007a). Im Gegensatz zum weitaus bekannteren und üblicherweise genutzten Extended Kalman Filter (EKF), welcher 
eine Linearisierungsmethode für die Bestimmung der $\mathrm{Zu}$ standsvariablen verwendet, basiert der UKF auf einem Regressionsansatz. Daher weist der UKF bei starken Nichtlinearitäten eine bessere Konvergenz mit identischem Rechenaufwand im Vergleich zum EKF auf (van der Merwe and Wan, 2004).

\section{Theoretische Grundlagen}

Dieser Absatz fasst die beiden Algorithmen des EKF und UKF kurz zusammen. Danach wird der Algorithmus des UKF dermaßen ergänzt, dass dieser verschiedene Nichtidealitäten des Sigma-Delta Wandlers schätzen kann. Dies beinhaltet im Besonderen die Modellierung des Sigma-Delta Wandlers und die Beschreibung des kombinierten UKF Algorithmus. Dabei schätzt der kombinierte UKF den $\mathrm{Zu}-$ standsvektor und die Parameter des Sigma-Delta Modulators. Dies wurde erstmalig in (Buhmann et al., 2007a) vorgestellt. Abschließend wird der Algorithmus beschrieben, welcher für die implizite Dezimation verwendet werden kann.

\subsection{Extended Kalman Filter}

Der klassische Ansatz mit einem Kalman Filter die $\mathrm{Zu}$ standsvariable $\boldsymbol{x}_{k}$ eines diskreten nichtlinearen Systems zu schätzen ist

$\boldsymbol{x}_{k}=f\left(\boldsymbol{x}_{k-1}, u_{1, k-1}, v_{k-1}\right)$

$y_{k}=h\left(\boldsymbol{x}_{k}, u_{2, k}, n_{k}\right)$.

Dabei ist $f$ die nichtlineare Zustandsfunktion und $h$ ist die nichtlineare Messfunktion. Die vorherige Prozessunsicherheit $v_{k-1}$ und das vorherige externe Eingangssignal $u_{1, k-1}$ treiben den Zustand des Systems voran und das Messrauschen $n_{k}$ erzeugt eine zusätzliche Unsicherheit bezüglich der Messung $y_{k}$. Der externe Eingang $u_{2, k}$ stellt ein bekanntes Eingangssignal dar, welches sich direkt auf die Messung auswirkt. Unter der Annahme, dass das Prozess- und Messrauschen einer Zufallsvariablen mit Normalverteilung folgt, ist der Kalman Filter die erste Wahl den unbekannten Zustand $\boldsymbol{x}_{k}$ des Systems rekursiv zu schätzen. Dies geschieht durch

$$
\begin{aligned}
\widehat{\boldsymbol{x}}_{k} & =\widehat{\boldsymbol{x}}_{k}^{-}-\mathbf{K}_{k}\left(y_{k}-\widehat{y}_{k}\right) \\
\mathbf{P}_{\boldsymbol{x}_{k}} & =\mathbf{P}_{\boldsymbol{x}_{k}}^{-}-\mathbf{K}_{k} \mathbf{P}_{\widehat{y}_{k}} \mathbf{K}_{k}^{T} .
\end{aligned}
$$

Dabei ist $\widehat{\boldsymbol{x}}_{k}$ der geschätzte Zustand des Systems und $\mathbf{P}_{\boldsymbol{x}_{k}}$ dessen Kovarianz, wie auch $\widehat{\boldsymbol{x}}_{k}^{-}$der vorhergesagt Zustand und $\mathbf{P}_{\boldsymbol{x}_{k}}^{-}$dessen Kovarianz. Die erwarteten Messwerte und deren Kovarianz sind gegeben durch $\widehat{y}_{k}$ und $\mathbf{P}_{\widehat{y}_{k}}$. Für den EKF ergeben sich dann für den vorhergesagten Zustand, die vorhergesagte Messung und die Kalman Verstärkung (Buhmann et al., 2007a)

$$
\begin{aligned}
& \widehat{\boldsymbol{x}}_{k}^{-} \approx f\left(\widehat{\boldsymbol{x}}_{k-1}, u_{1, k}, \bar{v}\right) \\
& \widehat{y}_{k} \approx h\left(\widehat{\boldsymbol{x}}_{k}^{-}, u_{2, k}, \bar{n}\right) \\
& \mathbf{K}_{k} \approx \mathbf{P}_{\boldsymbol{x}_{k} y_{k}}^{\operatorname{lin}}\left(\mathbf{P}_{\bar{y}_{k}}^{\operatorname{lin}}\right)^{-1}
\end{aligned}
$$

dabei sind $\bar{v}$ und $\bar{n}$ der Mittelwert des Prozess- und Messrauschens. Häufig werden diese Werte mit Null angenommen. Die verschiedenen, linearisierten Kovarianzen werden durch eine Reihenentwicklung der nichtlinearen Funktion bestimmt. Dabei wird die Reihenentwicklung häufig nach dem erster Glied abgeschnitten.

\subsection{Unscented Kalman Filter}

Wie im vorherigen Absatz erwähnt, basiert der EKF auf einer Reihenentwicklung der nichtlinearen Übertragungsfunktion am Punkt des vorhergesagten Mittelwerts. Im Gegensatz dazu bestimmt der UKF den Mittelwert und die Varianz mit Hilfe einer linearen Regression (van der Merwe and Wan, 2004; Julier et al., 1995) und ist daher eine ableitungsfreie Alternative zum EKF. Hierzu wird zunächst wie beim EKF angenommen, dass die verschiedenen Zufallsvariablen einer Normalverteilung folgen. Um auch hier (2) berechnen zu können, müssen zuerst die verschiedenen Sigma-Punkte $\mathbf{X}$ bestimmt werden durch

$$
\mathbf{X}_{k-1}=\left[\begin{array}{lll}
\widehat{\boldsymbol{x}}_{k-1} & \left.\widehat{\boldsymbol{x}}_{k-1}+\lambda \sqrt{\mathbf{P}_{k-1}} \widehat{\boldsymbol{x}}_{k-1}-\lambda \sqrt{\mathbf{P}_{k-1}}\right]
\end{array}\right]
$$

Die Sigma-Punkte bestehen einerseits aus dem vorherigen geschätzten Zustand $\widehat{\boldsymbol{x}}_{k-1}$, wie auch dessen Kovarianz $\mathbf{P}_{k-1}$. Zusätzlich wird noch ein Gewichtungsfaktor $\lambda$ eingeführt, um die Spreizung des Sigma-Punkte verändern zu können. Diese Sigma-Punkte stellen dann den wahren Mittelwert und Kovarianz dar. Im nächsten Schritt werden dann die SigmaPunkte $\mathbf{X}_{k-1}$ durch die nichtlineare Zustandsfunktion propagiert, um die zeitliche Änderung des Systemzustandes zu beschreiben

$\mathbf{X}_{k \mid k-1}=f\left(\mathbf{X}_{k-1}, u_{1, k-1}, v_{k-1}\right)$.

Während dieses Schrittes nehmen die erwarteten SigmaPunkte $\mathbf{X}_{k \mid k-1}$ den wahren zukünftigen Mittelwert $\widehat{\boldsymbol{x}}_{k}^{-}$und dessen Kovarianz $P_{\boldsymbol{x}_{k}}^{-}$auf. Diese können dann durch die lineare Regression bestimmt werden

$$
\begin{aligned}
\widehat{\boldsymbol{x}}_{k}^{-} & =\sum_{i=0}^{2 L} w_{i}^{m} \mathbf{X}_{i, k \mid k-1} \\
\mathbf{P}_{\boldsymbol{x}_{k}}^{-} & =\sum_{i=0}^{2 L} w_{i}^{c}\left(\mathbf{X}_{i, k \mid k-1}-\widehat{\boldsymbol{x}}_{k}^{-}\right)\left(\mathbf{X}_{i, k \mid k-1}-\widehat{\boldsymbol{x}}_{k}^{-}\right)^{T}
\end{aligned}
$$

Dabei sind $w_{i}^{m}$ und $w_{i}^{c}$ die linearen Regressionskoeffizienten für den Mittelwert und die Varianz. Diese Koeffizienten hängen einzig von dem verwendeten Sigma-Punkt Kalman Filter ab (Julier et al., 1995). Der Korrekturschritt auf Basis der Messungen wird in ähnlicher Weise durchgeführt. Hierzu werden wieder die erwarteten Sigma-Punkte $\mathbf{X}_{i, k \mid k-1}$ durch die Messgleichung propagiert, was zu den Messwert-SigmaPunkten $\mathbf{Y}_{k \mid k-1}$ führt. Dies lässt sich beschrieben durch

$\mathbf{Y}_{k \mid k-1}=h\left(\mathbf{X}_{k \mid k-1}, u_{2, k}, n_{k}\right)$. 
Von diesen Messwert-Sigma-Punkten lassen sich der erwartete Messwert $\widehat{y}_{k}$, die Messwertvarianz $\mathbf{P}_{\widehat{y}_{k}}$ und die Kovarianz zwischen den Messwert-Sigma-Punkten und erwarteten Sigma-Punkten berechnen durch

$$
\begin{aligned}
\widehat{y}_{k} & =\sum_{i=0}^{2 L} w_{i}^{m} \mathbf{Y}_{i, k \mid k-1} \\
\mathbf{P}_{\widehat{y}_{k}} & =\sum_{i=0}^{2 L} w_{i}^{c}\left(\mathbf{Y}_{i, k \mid k-1}-\widehat{y}_{k}\right)\left(\mathbf{Y}_{i, k \mid k-1}-\widehat{y}_{k}\right)^{T} \\
\mathbf{P}_{\boldsymbol{x}_{k}, y_{k}} & =\sum_{i=0}^{2 L} w_{i}^{c}\left(\mathbf{X}_{i, k \mid k-1}-\widehat{\boldsymbol{x}}_{k}^{-}\right)\left(\mathbf{Y}_{i, k \mid k-1}-\widehat{y}_{k}^{-}\right)^{T} .
\end{aligned}
$$

Letztendlich kann mit (8) nun (2) berechnet werden.

\subsection{Sigma-Delta System Modell}

Ein Modell des Sigma-Delta Wandlers ist in Abb. 1 dargestellt. Der Wandler besteht aus dem Schleifenfilter, welcher in einer Zustandsraumbeschreibung gegeben ist, einem Quantisierer und einem DAC. Da der Filteralgorithmus die Messwertdaten abgetastet erhält, empfiehlt es sich den zeitkontinuierlichen Filter mit einem zeitdiskreten Äquivalent zu beschreiben. Dadurch zeigt sich schon, dass Fehler, welche keine Auswirkung auf das zeitdiskrete Modell haben, auch nicht geschätzt werden können. Des Weiteren können auch Fehler, welche zu einer identischen diskreten Beschreibung führen, nicht von dem Filter unterschieden werden. Dies stellt aber keinen Nachteil des vorgestellten Verfahrens dar, da Fehler mit dieser Eigenschaft auch auf identische Art und Weise korrigiert werden können. Die Umwandlung des zeitkontinuierlichen Wandlers in einen zeitdiskreten Wandler kann durch die Anwendung der Lifting-Methode erreicht werden (Buhmann et al., 2007b). Der Sigma-Delta Wandler lässt sich dann beschreiben durch

$$
\begin{aligned}
\boldsymbol{x}_{k+1} & =\mathbf{A} \boldsymbol{x}_{k}+\mathbf{B}\left[u_{i n, k} D A C_{k}\right]^{T} \\
y_{\text {out }, k} & =Q\left(\mathbf{C} \boldsymbol{x}_{k}+\mathbf{D} u_{k}+p_{k}\right) .
\end{aligned}
$$

Dabei stellen A, B, C, und $\mathbf{D}$ die Systemmatrix, Steuermatrix, Beobachtungsmatrix und Durchgangsmatrix dar. Das Gesamtsystem, welches aus Sigma-Delta Modell und dem kombinierten UKF (Buhmann et al., 2007a) besteht wurde in der ReBEL Toolbox (van der Merwe and Wan, ReBEL) in MATLAB ${ }^{\mathrm{TM}}$ modelliert. Basierend auf (9) und unter der Annahme, dass die vorherigen DAC Werte $D A C_{k-1}$ bekannt sind, können die notwendigen Gleichungen für die Implementierung in der ReBEL Toolbox sehr einfach abgeleitet werden. Diese sind

$$
\begin{aligned}
\text { ffun }= & \mathbf{A} \boldsymbol{x}_{k-1}+\mathbf{B}_{i n} v_{k-1}+ \\
& \mathbf{B}_{D A C} D A C_{k-1} \\
\text { hfun }= & Q\left(\mathbf{C} \boldsymbol{x}_{k}+\mathbf{D} u_{k}+p_{k}\right)+n_{k} .
\end{aligned}
$$

dabei stellen ffun und hfun die Zustandsfunktion und die Messfunktion dar. $\mathbf{B}_{\text {in }}$ und $\mathbf{B}_{D A C}$ sind die Spalten der B Ma-

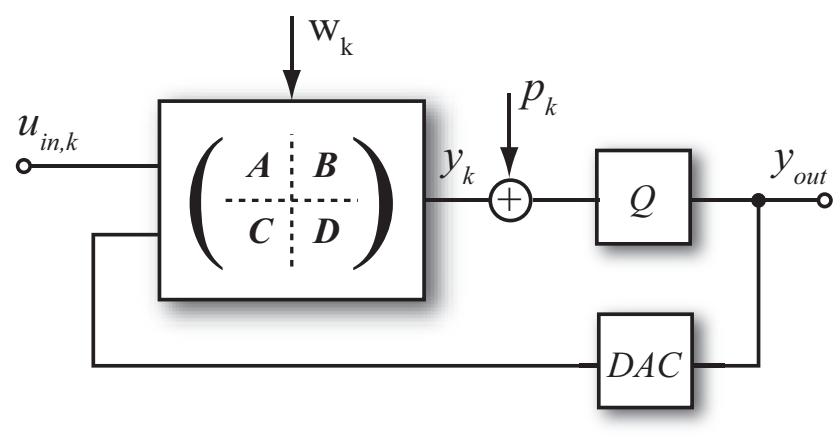

Abb. 1. Zustandsraum Beschreibung eines zeitdiskreten SigmaDelta Wandlers, welcher aus dem Filter, einem Quantisierer $Q$ und einem DAC besteht. Dem Quantisierer Eingang wird zur Verbesserung der Schätzung ein bekanntes pseudozufälliges Rauschen $p_{k}$ hinzugefügt.

trix, welche die Steuermatrix des Sigma-Delta Wandlers darstellt. Die Implementierung des EKF ist von der für den UKF vorgestellten Herangehensweise leicht unterschiedlich. Um ein implementierbares Modell zu erzeugen, muss zunächst der Quantisierer $Q$ linearisiert werden. Hierzu findet man unterschiedliche Ansätze in der Literatur. Die bekannteste Methode ist eine signalabhängige Verstärkung mit additivem weißem Rauschen. Die verschiedenen EKF Matrizen, $\mathbf{A}_{\mathrm{EKF}}$, $\mathbf{B}_{\mathrm{EKF}}, \mathbf{C}_{\mathrm{EKF}}, \mathbf{D}_{\mathrm{EKF}}, \mathbf{G}_{\mathrm{EKF}}, \mathbf{H}_{\mathrm{EKF}}, \mathbf{J F} \mathbf{W K F}_{\mathrm{EKF}}$ und $\mathbf{J H} \mathbf{W}_{\mathrm{EKF}}$, lassen sich dann durch die Differentation von (10) und (11) nach den verschiedenen Variablen (van der Merwe and Wan, ReBEL), wie z.B. dem Zustandsraum oder den Modellparametern, bestimmen.

\subsection{Implizite Dezimation}

Die implizite Dezimation des Sigma-Delta Ausgangs mit einem UKF Filter ähnelt dem Ansatz der Dezimation mit einem EKF (Abeysekera and Charoensak, 2006). Hierzu muss der Filteralgorithmus aus Abschnitt 2.3 angepasst werden. Dies geschieht durch Hinzufügen von weiteren Zustandsvariablen zum Zustandsraum. Diese zusätzlichen Zustandsvariablen werden dann für die Beschreibung des Wandlereingangs $u_{k}$ verwendet. Falls man ein konstantes Eingangssignal zwischen zwei Zeitschritten annimmt und deren Abweichung sich durch eine Normalverteilung annähern lässt, reicht eine einzige zusätzliche Zustandsvariable aus. Folglich ergibt sich dann für den modifizierten UKF Algorithmus

$$
\begin{aligned}
\boldsymbol{x}_{i n, k}= & \boldsymbol{x}_{i n, k-1}+v_{k-1} \\
\text { ffun }= & \mathbf{A} \boldsymbol{x}_{k-1}+\mathbf{B}_{i n} \boldsymbol{x}_{i n, k}+ \\
& \mathbf{B}_{D A C} D A C_{k-1} \\
\text { hfun }= & Q\left(\mathbf{C} \boldsymbol{x}_{k}+\mathbf{D} u_{k}+p_{k}\right)+n_{k}
\end{aligned}
$$

In (12) stellt $\boldsymbol{x}_{i n, k}$ die zusätzliche Zustandsraumvariable dar, welche den Eingang des Wandlers $u_{i n, k}$ repräsentiert. Um aber eine bessere und stärker geglättete Schätzung zu 


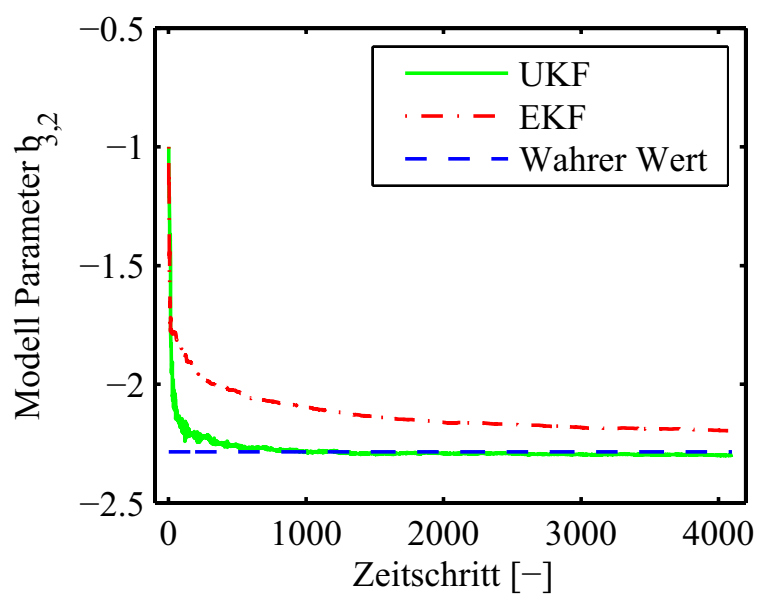

Abb. 2. Parameter Schätzung durch den UKF und EKF Algorithmus. Dies zeigt die weitaus besseren Konvergenzeigenschaften des UKF.

erhalten, kann eine Reihenentwicklung höherer Ordnung für den Eingang des Wandlers verwendet werden. Dies führt aber auch zu einem höheren Rechenaufwand. Die Dezimation des Wandlerausgangs (Abeysekera and Charoensak, 2006) findet durch rekursive Ausführung von (12) statt. Alle $N$ Schritte, dabei ist $N$ identisch zum Überabtastungsverhältnises (OSR), wird dann der dezimiert Ausgang $u_{i n, n \times O S R}$ aus dem Datenstrom entnommen. Danach muss der Filter neu initialisiert werden. Dies beinhaltet den Zustandsraum und die Kovarianzen des Filters. Diese Prozedur realisiert die Tiefpassfilterung des Eingangssignals. Im Falle des EKF Dezimationsfilters kann gezeigt werden, dass der Filterfehler die gleiche Ordnung aufweist, wie ein optimaler FIR Filter mit einer gewählten Filterordnung von $N$. Der Grund hierfür ist, dass der EKF im nichtstationären Fall einem Wiener Filter entspricht. Da der UKF und EKF selbst ähnliches Verhalten aufweisen, sollte der UKF auch diese Eigenschaft aufweisen. Dies gilt es aber noch zu beweisen.

\section{Simulationsergebnisse}

In diesem Abschnitt werden verschiedene Simulationsergebnisse des gekoppelten UKF gezeigt. Diese bestätigen die prinzipielle Funktion der Zustandsraumschätzung, der Parameterschätzung und der impliziten Dezimation. Dies wird anhand eines 3er Ordnungs Sigma-Delta Wandler mit 4 Bit Quantisierer gezeigt. Der UKF und der EKF sind implementiert, wie in Abschnitt 2 beschrieben. Die zusätzlichen Filterparameter sind aus (Buhmann et al., 2007a) entnommen. Um die EKF Simulation zu vereinfachen, wurde eine Quantisiererverstärkung $K_{q}$ von 1 angenommen. Diese Annahme wird häufig bei Multi-Bit Sigma-Delta Wandler angewendet, falls keine Variation in den Koeffizienten vorhanden ist. Unter die-

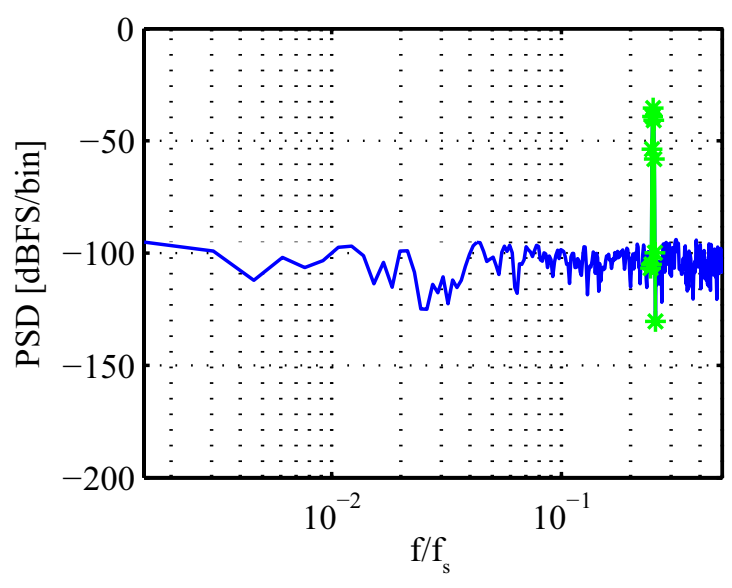

Abb. 3. Leistungsdichtespektrum des geschätzten Eingangs $\boldsymbol{x}_{i n}$ mit einer Signalamplitude von $-36 \mathrm{dBFS}$ und einer Signalfrequenz, welche bei der Hälft der Signalbandbreite nach der Dezimation liegt.

ser Annahme vereinfacht sich der EKF zu einem klassischen Kalman Filter.

\subsection{Sigma-Delta Wandler}

Der Sigma-Delta Wandler wurde mit der DISCO Toolbox (Buhmann et al., 2007b) synthetisiert. Als OSR wurde $O S R=25$ gewählt. Die maximale Anzahl an Quantisiererstufen, welche vom rückgekoppelten Quantisierungsrauschen verwendet wird, wurde eins größer als die Ordnung des Modulators gewählt. Dadurch ergibt sich eine maximale stabile Eingangsamplitude von $62.5 \% V_{\text {ref }}$ (Schreier and Temes, 2004). Der berechnete Schleifenfilter des Sigma-Delta Wandlers ergibt sich dann zu

$$
\left(\begin{array}{c|c}
\mathbf{A} & \mathbf{B} \\
& \\
\hline \mathbf{C} & \mathbf{D}
\end{array}\right)=\left(\begin{array}{rrr|rr}
1 & 0 & 0 & 0.55 & -0.55 \\
1 & 1 & 0 & 0 & -1.61 \\
0 & 1 & 1 & 0 & -2.04 \\
\hline 0 & 0 & 1 & 0 & 0
\end{array}\right) .
$$

3.2 Schätzung von Modellparametern und implizite Dezimation

Abbildung 2 zeigt die Simulationsergebnisse des gekoppelten UKF und des gekoppelten EKF. Dabei sollten die Filter den Modellparameter $b_{3,2}$ der Steuermatrix B schätzen. Bei beiden Simulationen wurde der Zustandraums und die dazugehörigen Kovarianz mit gleichen Startwerten initialisiert. Um die Konvergenz beider Algorithmen besser vergleichen zu können, wurde zusätzlich ein großer Fehler zum wahren Wert hinzugefügt. Wie die Abbildung zeigt, besitzt der UKF eine bessere Konvergenzeigenschaft im Vergleich zum EKF, da dieser schneller zum wahren Wert strebt. Nichtsdestotrotz 
streben beide Filter, UKF und EKF, dem wahren Wert entgegen. In Abb. 3 ist das dezimierte Spektrum der Zustandsraumvariablen $\boldsymbol{x}_{i n, k}$ nach dem Heruntertasten zur Nyquist Abtastrate dargestellt. Dies bestätigt die implizite Dezimation des vorgestellten Algorithmus, da ansonsten die Rauschdichte einen weitaus höheren Wert annehmen müsst.

\subsection{Schätzung von Sensorparametern in einem kraftkom- pensierten Sigma-Delta Wandler}

Ein im Vergleich zu den elektrischen Sigma-Delta Wandlern noch recht junges Themengebiet, sind so genannte kraftkompensierte Sigma-Delta Wandler, bei denen der Sensor selbst ein Teil des Sigma-Delta Regelkreises ist. Dies ist in Abb. 4 dargestellt. Ein großes Problem elektromechanischer Sigma-Delta Wandler ist, dass die Variation der Sensorparameter unabhängig von der Variation der elektrischen Filter geschieht. Dadurch wird eine Anpassung der Filterstruktur an das verwendete Sensorelement unumgänglich, um einen stabilen Betrieb zu gewährleisten. Das hierfür benötigte Modell kann sehr einfach aus dem des elektrischen Sigma-Delta Wandler (11) abgeleitet werden. Hierfür muss dieses einfach um das mechanische Element ergänzt werden. Dadurch ergibt sich für das neue Modell des Schleifenfilters

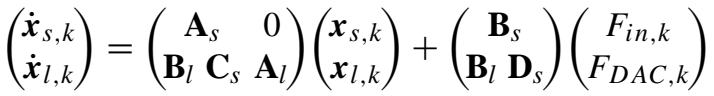

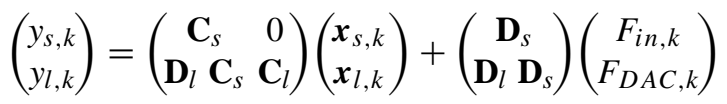

Aus (14) lassen sich in einem nächsten Schritt die verschiedenen Funktionen für den UKF und EKF ableiten, wie in Abschnitt 2.3 vorgeschlagen.

\section{Diskussion}

Es wurde eine Methode zur Schätzung von Schaltungsnichtidealitäten und Sensorparametern vorgestellt. Dies ist ein wichtiger Schritt, um eine Korrektur in Echtzeit zukünftig durchführen zu können. Die Effizienz dieser Methode kann durch Einbeziehung der Dezimation mit in den Filter verbessert werden. Dadurch entfällt der ansonsten zusätzlich benötigte Dezimationsfilter. Es wurden Simulationsergebnisse gezeigt, welche die Konvergenzeigenschaft und die implizite Dezimation betrachten. Natürlich ist der vorgestellte Ansatz nicht so effizient wie ein Polyphasen Filter oder ein FIR Filter bezüglich der Dezimation. Aber der vorgestellte Ansatz ermöglicht die gleichzeitige Schätzung von Schaltungsnichtidealitäten. Vergleicht man den EKF und UKF miteinander, zeigen beide Methoden eine ähnliche Effizienz. Der UKF benötigt aber keine Linearisierung des Quantisierers $Q$. Daher ist weder ein zusätzliches Wissen über die Quantisiererverstärkung nötig, noch muss dieser separat geschätzt werden.

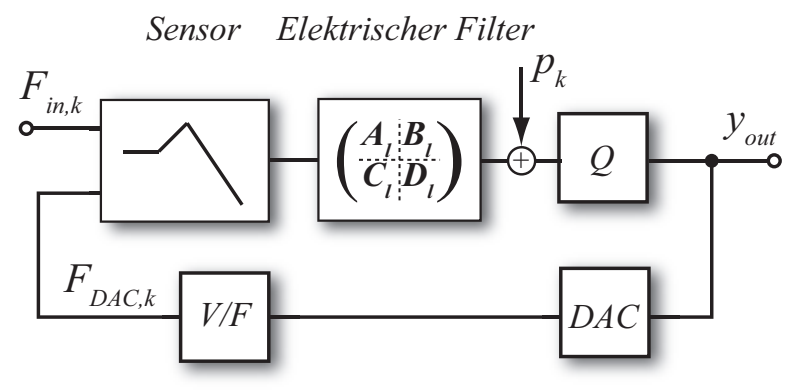

Abb. 4. Kraftkompensiertes Auswertesystem basierend auf einem Sigma-Delta Wanlder, welches aus einem Sensorelement, einem elktrischen Filter, einem Quantisierer $Q$, einem DAC und einem Wandlerelement $V / F$ besteht.

Danksagung. Teile dieser Arbeit wurden im Rahmen des Projektes „Entwicklung eines Mikrosystems mit niedriger Verlustleistung unter Verwendung der innovativen Sigma-Delta Technik (AiF-No. 205 $\mathrm{ZN}$ )“ erstellt. Das Projekt wird gefördert durch das BMWi, welches sich über das BMBF finanziert.

\section{Literatur}

Abeysekera, S. S. and Charoensak, C.: Efficient Realization of Sigma-Delta $(\Sigma-\Delta)$ Kalman Lowpass Filter in Hardware Using FPGA, EURASIP Journal on Applied Signal Processing, vol. 2006, article no. 52736, 11 pp., 2006.

Balestrieri, E., Daponte, P., and Rapuano, S.: A state of the art on ADC error compensation methods, Instrumentation and Measurement Technology Conference, 1, 711-717, 2004.

Buhmann, A., Keller, M., Ortmanns, M., and Manoli, Y.: An Unscented Kalman Filter for the Estimation of Circuit Nonidealities with Implicit Decimation in Continuous-Time Multibit SigmaDelta Modulators, IEEE International Midwest Symposium on Circuits and Systems, Montreal, Canada, 2007a.

Buhmann, A., Keller, M., Ortmanns, M., and Manoli, Y.: DISCO - A Toolbox for the Discrete-Time Simulation of ContinuousTime Sigma-Delta Modulators Using MATLAB ${ }^{\text {TM }}$, IEEE International Midwest Symposium on Circuits and Systems, Montreal, Canada, 2007b.

Cauwenberghs, G. and Temes, G. C.: Adaptive digital correction of analog errors in MASH ADCs - Part I: Off-line and blind on-line calibration, IEEE Trans. Circuits Syst. II, 47(7), 621-628, 2000.

Julier, S., Uhlmann, J., and Durrant-Whyte, H.: A new approach for filtering nonlinear systems, in: Proceedings of the American Control Conference, 1628-1632, 1995.

Schreier, R. and Temes, G. C.: Understanding Delta-Sigma Data Converters, New York: Wiley, 2004.

van der Merwe, R. and Wan, E. A.: Sigma-Point Kalman Filters for Integrated Navigation, in: Proceedings of the 60th Annual Meetings of The Institute of Navigation (ION), Dayton, OH, June, 2004.

van der Merwe, R. and Wan, E. A.: ReBEL : Recursive Bayesian Estimation Library, available at: http://choosh.csee.ogi.edu/ rebel/, 2008. 